\title{
ADRIEN QUÉRET-PODESTA
}

Académie polonaise des Sciences, Institut d'études slaves, Département d'Histoire

\section{Les manuscrits islandais médiévaux dans les romans islandais contemporains : l'exemple de L'Énigme de Flatey ${ }^{1}$}

\author{
Medieval Icelandic Manuscripts: \\ The Case of The Flatey Enigma
}

\begin{abstract}
In the rich history of Icelandic literature, the most famous literary genres are undoubtedly the medieval sagas and the contemporary criminal novels. However, those genres are as not as far from each other as one may think, since masterpieces of Icelandic medieval literature are sometimes summoned by contemporary authors, as is shown in The Flatey Enigma (Icelandic: Flateyjargáta), a criminal novel by Viktor Arnar Ingólfsson which is built around the story and the contents of the Book of Flatey, a famous fourteenth Icelandic manuscript. The present article provides an analysis of the place and function of the manuscripts and the medieval texts it contains: the results obtained show that their main function is to help the development of the plot, although some intertextual references also have a didactic dimension, whereas others provide information about the relations between the characters and the Book of Flatey.
\end{abstract}

KEY WORDS: Criminal novels, Iceland, manuscripts, Viktor Arnar Ingólfsson

\section{Introduction}

Dans la longue et riche histoire de la littérature islandaise, les genres les plus connus du grand public sont certainement les sagas et poèmes épiques mé-

${ }^{1}$ Viktor Arnar Ingólfsonn, L'Énigme de Flatey, traduit par Patrick Guelpa, Seuil, Paris, 2013. Texte original : Flateyjargáta. Mál og menning, Reykjavík, 2002. En raison de notre méconnaissance de l'islandais, nous avons, sauf mention contraire, utilisé les traductions françaises des œuvres citées dans le cadre de la présente étude. 
diévaux ainsi que les polars et romans noirs contemporains. Ces deux genres ne sont d'ailleurs pas si éloignés que l'on pourrait le croire, puisque les chefsd'œuvre de la littérature islandaise du Moyen Âge sont parfois convoqués par les auteurs actuels, comme l'illustre notamment le cas du roman policier L'Énigme de Flatey, de Viktor Arnar Ingólfsson. L'intrigue de cet ouvrage est ainsi centrée autour du Livre de Flatey, célèbre manuscrit islandais réalisé au XIV e siècle et comportant pas moins de 80 textes différents.

L'intrigue de L'Énigme de Flatey se déroule en une semaine, du mercredi $1^{\text {er }}$ août 1960 au mercredi 8 août 1960 ; elle a lieu essentiellement sur l'île de Flatey, dans le Breiðafjörður, au nord-ouest de l'Islande ${ }^{2}$, mais certaines scènes ont pour cadre les îlots environnants ou encore la capitale du pays, Reykjavik. Le roman débute d'ailleurs par la découverte d'un cadavre sur l'île inhabitée de Ketilsey, au sud de Flatey; le défunt, qui se nommait Gaston Lund, était un professeur de l'Université de Copenhague venu sur l'île pour essayer de résoudre la solution de l'«énigme de Flatey». Cette énigme est une devinette très élaborée composée d'une série de questions sur le contenu du Livre de Flatey, un célèbre manuscrit médiéval islandais rassemblant de nombreuses sagas et autres récits qui doit son nom au fait qu'il fut conservé un temps à Flatey avant d'être emporté au Danemark; il y est encore à l'époque du récit ${ }^{3}$, mais une édition en fac-similé contenant les feuilles volantes sur laquelle est écrite le texte de l'énigme se trouve dans la petite bibliothèque de l'île. Kjartan, un employé de la préfecture de Patreksfjörður, est chargé de l'enquête sur place; il est aidé dans sa tâche par plusieurs insulaires dont Grímur et Högni, respectivement bourgmestre et instituteur de l'île, mais aussi de la doctoresse Johanna Thorvald à laquelle il est lié de manière tragique puisqu'il est en partie responsable de la mort de son fiancé, Einar, ce qui lui valut plusieurs années de prison -, mais le mystère s'épaissit encore avec la découverte d'un second cadavre mutilé selon un ancien rituel viking et abandonné dans le cimetière de l'île.

Le présent article a pour but de proposer un essai d'analyse de la réception du Livre de Flatey et des textes qu'il contient dans L'Énigme de Flatey de Viktor Arnar Ingólfsson. Afin de mener à bien cet objectif, nous analyserons tout d'abord la place et l'usage des textes islandais médiévaux évoqués dans le roman à travers le prisme de l'intertextualité, puis nous nous intéresserons au rôle du manuscrit en tant qu'objet symbolique avant de nous interroger sur le rapport entretenu par les personnages des romans d'Ingólfsson et d'Indridason avec le manuscrit et les textes qu'il contient.

${ }^{2}$ Ces précisions ne sont pas inutiles car il existe en réalité deux îles nommées Flatey (l'île plate, en islandais) au large des côtes islandaises; la seconde île se trouve près de la côte septentrionale de l'Islande.

${ }^{3}$ Le Livre de Flatey et plusieurs autres célèbres manuscrits islandais ont été rendus à l'Islande par le Danemark en 1971. 


\section{La place des textes islandais médiévaux dans L'Énigme de Flatey}

Bien que L'Énigme de Flatey de Viktor Arnar Ingólfsson contienne quelques brèves références à des œuvres littéraires (essentiellement islandaises ou scandinaves) $\mathrm{du} \mathrm{XX}^{\mathrm{e}}$ siècle et à des journaux, la très grande majorité des cas d'intertextualité présents dans ce roman concernent des textes islandais médiévaux, qui sont évoqués en 67 occasions. Une analyse détaillée des œuvres employées démontre que 62 des 67 occurrences - soit plus de $90 \%$ - proviennent de textes contenus dans le Livre de Flatey, ce qui s'explique naturellement par la présence de l'«énigme de Flatey» dans cette œuvre. Le récit de la résolution de cette énigme, qui se déroule durant la nuit du dimanche 5 juin au lundi 6 juin, est divisé en fragments situés à la fin de chaque chapitre du roman; rapporté sous la forme d'un dialogue entre Johanna et Kjartan, il commence par un long monologue dans lequel la doctoresse donne une longue description de la genèse, $\mathrm{du}$ contenu et de l'histoire (quatorze fragments) et se poursuit par trente neuf questions, qui permettent chacune de trouver une lettre alors que la $40^{\mathrm{e}}$ question délivre pour sa part la clé d'un code qui permet d'accéder, en plusieurs étapes, à la réponse de l'énigme. La narration de la résolution de l'« énigme» constitue donc ainsi une histoire dans l'histoire en même temps qu'elle constitue la raison d'être du roman tout entier: en effet, c'est la présence de l'énigme sur l'île qui entraîne la venue de Gaston Lund à Flatey, un évènement dont les conséquences seront notamment sa mort et celle de son fils caché, Bryngeir. Cette devinette savante constitue donc le cœur du récit et l'on peut penser que c'est l'importance de ce texte fictif qui explique pourquoi ce dernier, et non pas le manuscrit qui en est l'objet, a donné son nom au roman, bien que l'hypothèse d'une volonté de jouer sur les différentes interprétations du mot "énigme» pour créer une allusion au mystère représenté par les deux décès tragiques survenus à Ketilsey et Flatey ne puisse être totalement exclue.

Le récit de la résolution de l'« énigme de Flatey » en elle-même contient environ deux tiers des cas d'intertextualité provenant du Livre de Flatey (42/62) et plus de $60 \%$ du total des références à des œuvres médiévales; on peut encore ajouter à ce chiffre deux mentions de la Heimskringla, ou Saga des rois de Norvège, une énumération de cinq œuvres du Livre de Flatey et trois proverbes cités par Johanna dans sa description de ce manuscrit précédant la résolution de l'énigme, ce qui donne pour cette seule narration enchâssée dans le roman 52 références textuelles sur 67 , soit plus de trois quarts de l'œuvre. Le fait que le chiffre de références, à savoir 42 , ne soit pas identique au nombre de questions, c'est-à-dire 40, s'explique par la présence de deux références en deux occasions : ainsi, le père de Johanna, Björn Snorri Thorvald, et l'universitaire danois Gaston Lund, dont les réponses diffèrent en plusieurs occasions, ne sont pas d'accord sur l'œuvre d'où vient la réponse à la troisième question (Saga des Groenlandais 
pour le premier, tandis que le second penche visiblement pour la Saga d'Olaf Tryggvason) alors que la résolution finale de l'énigme contient une citation de la Saga de Håkon l'ancien et une autre de la Saga de Sverrir (réponse finale).

Les cas d'intertextualité concernant les œuvres contenues dans le Livre de Flatey semblent essentiellement provenir d'une petite quinzaine de textes, mais le fait que la provenance de certains fragments est difficile à établir suggère que ce chiffre pourrait être plus élevé : ce total demeure cependant relativement modeste au regard du nombre total d'œuvres contenues dans le manuscrit, qui se compose d'environ 80 textes différents. La très grande majorité de ces œuvres sont des sagas et des «dits» (en islandais paettir ${ }^{4}$ ), mais le manuscrit contient également quelques autres textes, comme des annales, qui sont évoquées dans la description du Livre de Flatey par Johanna. Le nombre de sagas et de dits évoqués dans le roman forme un certain contraste avec la composition du manuscrit, puisque la liste des textes identifiés contient neuf sagas (Saga d'Olaf Tryggvason, Saga de Saint Olaf, Saga de Sverrir, Saga de Håkon l'Ancien, Saga des vikings de Jómsborg, Saga des Groenlandais, Saga des frères jurés, Saga de Magnus et Saga des Orcadiens) sur un total de treize dans le Livre de Flatey mais elle ne comporte que quatre dits (Dit de Sneglu Halli, Dit de Sörli, Dit de Norna-Gestr et Dit d'Ormur Stórólfson) alors que le manuscrit en contient une cinquantaine: cette différence peut s'expliquer par le fait que les dits sont parfois intégrés aux sagas, mais aussi par la plus grande popularité des sagas auprès du grand public.

Cette notion de popularité est peut-être aussi à l'origine des références aux quatre œuvres ne figurant pas dans le Livre de Flatey, à savoir la Heimskringla, la Saga de Grettir, la Saga d'Egil, fils de Grimr le Chauve et la Saga de Njáll le Brûlé. En plus de leurs fonctions spécifiques, la présence de références à ces trois œuvres dans les deux romans s'explique peut-être par leur grande popularité : ainsi, la Heimskringla, ou Saga des rois de Norvège, compilation de sagas royales (à l'exception de la Saga des Ynglingar, placée au début de l'œuvre) réalisée par le célèbre écrivain islandais Snorri Sturluson ${ }^{5}$, auquel on attribue également la rédaction de la Saga d'Egil, fils de Grimr le Chauve et parfois celle de la Saga de Njáll le Brûlé, est l'une des œuvres les plus importantes de la littérature islandaise médiévale. L'analyse conduite par Emily Lethbrige démontre en outre que la Saga de Njáll le Brûlé et la Saga d'Egil, fils de Grimr le Chauve sont les deux sagas des Islandais apparaissant le plus souvent dans les manuscrits islandais antérieurs à la Réforme (Lethbridge, 2014: 66) et la chercheuse de Cambridge affirme également que la Saga de Njáll le Brûlé est «la plus connue

${ }^{4}$ Un páttr (pluriel paettir) est un récit en ancien islandais: les paettir sont généralement plus courts que les sagas.

${ }^{5}$ Né en 1179 et mort en 1241, Snorri Sturlurson s'est distingué par son activité littéraire (outre les textes mentionnés ci-dessus, nous lui devons également une Edda en prose) et politique. 
et la plus estimée » des sagas des Islandais ${ }^{6}$ (2014 : 55). Quant à la Saga de Grettir, elle apparaît également dans la catégorie des sagas le plus souvent recopiées avant la réforme dans l'étude d'Emily Leithbridge (2014: 66) et son principal protagoniste est évoqué dans plusieurs autres sagas islandaises : la cause de cet intérêt est sans doute liée au fait qu'il vécut proscrit pendant 19 ans, ce qui est la plus longue durée de survie pour un hors-la-loi dans l'ancienne Islande?.

Par ailleurs, une analyse détaillée de l'ensemble des sagas contenues dans le Livre de Flatey et mentionnées dans le roman permet de constater que cinq sont des sagas royales alors que deux autres (Saga des vikings de Jómsborg et Saga des Orcadiens) sont parfois classées dans cette catégorie et que les deux dernières sont des sagas des Islandais; si l'on inclut les sagas ne figurant pas dans le manuscrit, le nombre de sagas royales passe à six alors que celui des sagas des Islandais monte à cinq, ce qui atténue l'écart entre ces deux. Cette répartition par genres correspond à la composition du Livre du Flatey, qui ne comprend que ces deux types de sagas, mais elles reflètent également leur grande popularité parmi l'ensemble des sagas islandaises.

\section{Intertextualité et fonction des textes islandais médiévaux dans L'Énigme de Flatey}

En nous appuyant sur la classification des formes d'intertextualité proposée par Gérard Genette (Genette, 1982: 8) et en l'adaptant aux spécificités de L'Énigme de Flatey, nous pouvons constater que ce roman contient 58 citations, parmi lesquelles il faut distinguer 47 citations de textes, 21 citations de titre d'œuvres littéraires, dont environ la moitié sont associées à d'autres formes d'intertextualité, et 1 citation de genre littéraire; le récit de Viktor Arnar Ingólfsson contient également 45 cas de reformulation ou de paraphrase, lesquels sont associés à des citations en 41 occasions, ainsi que 3 allusions onomastiques créées à partir de noms de personnages et/ou d'auteurs de sagas, dont deux sont accompagnés.de citations de titres d'œuvres littéraires.

Comme dans le cas de la nature des œuvres citées, la fréquente association des citations et des cas de reformulations ou de paraphrases au sein de L'Énigme de Flatey résulte de la présence du jeu littéraire qui a donné son nom

${ }^{6}$ En islandais Íslendingasögur. Les spécialistes distinguent en effet plusieurs grandes familles au sein des sagas scandinaves. La classification établie par le grand spécialiste Sigurður Nordal en 1953 distingue ainsi cinq familles : les sagas royales, les sagas des Islandais, les sagas des contemporains, les sagas des chevaliers et les sagas légendaires. Par la suite, une sixième catégorie, celle des sagas des évêques, a été ajoutée.

${ }^{7}$ On en trouve notamment une mention dans la Saga de Gísli Súrsson. 
au roman. En effet, tous ces cas d'association figurent dans le récit de la résolution de l'énigme, dont les réponses, présentes à la fin des chapitres du roman, se composent d'une reformulation de l'épisode dans laquelle figure la réponse à chaque question ainsi que d'une citation du texte original : cette dernière est généralement assez courte et se limite parfois au mot contenant la lettre devant servir, grâce à l'usage d'un code, à trouver la solution de l'énigme. Par ailleurs, cinq réponses mentionnent aussi le titre de l'œuvre dont est titrée la solution à la question: les cinq œuvres mentionnées sont la Saga des Vikings du Jómsborg ( $1^{\text {re }}$ question), la Saga des Groenlandais ( $3^{\mathrm{e}}$ question), le Dit de Sörli (6 ${ }^{\mathrm{e}}$ question), la Saga de Håkon l'ancien (40 question) et la Saga de Sverrir (réponse finale). En raison de cette faible proportion de textes dûment identifiés, le nombre de cas où chaque lecteur est en mesure d'identifier l'œuvre dont provient la réponse à chaque question dépend naturellement du niveau de ses connaissances sur la littérature islandaise médiévale, mais l'identification de ces œuvres n'est pas nécessaire pour comprendre le déroulement de la narration concernant cette énigme.

Le récit de la résolution de l'«énigme de Flatey» au sens strict (c'est-à-dire sans le prologue consacré à la description du Livre de Flatey) contient donc pas moins de 42 citations et 41 cas de paraphrase, soit presque $80 \%$ des occurrences de chacune de ces formes d'intertextualité dans le roman; une telle proportion montre clairement que leur première fonction dans L'Énigme de Flatey est de faire avancer la résolution d'un des mystères de l'intrigue du roman. Afin de mettre ce résultat en perspective, il convient désormais de se pencher sur la fonction des autres cas d'intertextualité présents dans le roman. Pour ce faire, nous allons commencer par les cas de citation et de paraphrase ne figurant pas dans le récit concernant la résolution de l'«énigme de Flatey» stricto sensu: ce groupe comprend un total de neuf éléments, parmi lesquels on remarque 5 citations et 4 cas de paraphrases d'épisodes contenus dans les textes figurant dans le Livre de Flatey.

Le premier cas de citation intervient lors de la découverte du rapatriement du cadavre de Gaston Lund, puisque Grímur, le bourgmestre de l'île, récite une phrase de la Saga de Grettir: «ça ne sent pas très bon, fit Grettir le Fort en pénétrant dans le tertre» (Ingólfsson, 2013: 49). Quelques chapitres plus loin, Johanna explique à Kjartan qu'elle admire "les annalistes islandais » et cite en guise d'exemples trois proverbes figurant dans le Livre de Flatey et passés dans le langage courant: "Des phrases comme "La bière est un autre homme", "C'est toujours le plus sage qui cède", "Les fêtes font du bien"» (2013: 71).

La dernière citation provient de la Saga des Vikings du Jomsborg: vraisemblablement écrite au début du XIII siècle, cette œuvre relate les hauts faits d'un groupe de guerriers établis dans la forteresse de Jómsborg, un toponyme également utilisé dans la Scandinavie médiévale pour designer la localité de Wolin, située sur l'île du même nom, au nord-ouest de la Pologne (Morawiec, 
2017 : 135). Dans le roman, ce récit a inspiré la création d'un club d'étudiants appelé «Les Cousins des Vikings du Jómsborg» ou l'« Association des Vikings du Jómsborg» (Ingólfsson, 2013: 312), dont faisaient partie Bryngeir, Kjartan et Einar, le défunt fiancé de Johanna. Ainsi que l'expliquent Johanna et Kjartan au cours de leurs interrogatoires respectifs, les membres du club devaient subir une épreuve initiatique, à savoir un simulacre de décapitation à l'épée inspiré par un épisode tiré du récit de l'exécution des vikings du Jómsborg dans la saga du même nom: en effet, le récit rapporte que Svein Buáson retira sa tête du billot avant le coup fatal et fut finalement gracié. Dans le roman, la scène n'a pas une fin aussi heureuse puisque le nouvel entrant, Einar, est décapité par Kjartan après que Bryngeir, jaloux du fiancé de Johanna, a mis son genou dans le dos de ce dernier pour l'empêcher de reculer, ce qu'il n'avouera que des années plus tard à la doctoresse après avoir laissé Kjartan assumer seul la responsabilité du drame. Durant son interrogatoire, Kjartan rapporte que la lecture du récit de l'exécution des vikings du Jómsborg d'après la saga homonyme faisait partie de la cérémonie; il cite les deux phrases de la saga qui décrivent les préparatifs de l'exécution manquée de Svein Buáson et précise que les mots «frappe vite» sont le signal pour le «bourreau», qui doit alors abattre son épée sur le billot (2013: 330).

Si Johanna précise que les trois proverbes sont utilisés «à tout bout de champ» (2013: 71), les deux autres citations possèdent un contexte bien déterminé : ainsi le bourgmestre cherche-t-il à masquer son trouble en citant un passage de saga approprié aux circonstances alors que le fragment de la Saga des Vikings du Jomsborg est utilisé comme texte de cérémonie. Ces cinq cas de citations suggèrent que les enseignements du Livre de Flatey peuvent être utiles dans différentes situations: elles fournissent donc des informations sur le rapport que les personnages entretiennent avec les textes contenus dans le manuscrit, mais les trois citations de proverbes par Johanna possèdent également une fonction didactique, en particulier pour le lecteur islandais.

Les quatre cas de paraphrases de textes figurant dans le Livre de Flatey apparaissent seulement dans deux passages de L'Énigme de Flatey, à savoir les interrogatoires respectifs de Sigurbjörn et de Johanna par les policiers Pórólfur et Lukás; les textes utilisés relatent d'ailleurs tous des récits de morts brutales, ce qui explique leur étroite association à l'enquête. Ainsi, durant son interrogatoire, le fermier Sigurbjörn demande à Pórólfur s'il le croit vraiment capable de découper un «aigle de sang» dans le dos de Bryngeir : devant l'incompréhension du policier, il raconte le récit de ce supplice dans le Dit de Norna-Gestr et ajoute que la Saga des Orcadiens et le Dit d'Ormur Stórólfson l'évoquent également (2013 : 275). Sigurbjörn dresse également une analogie entre la mort de Gaston Lund et le récit d'Eyvindur Kelda, qui avait voulu tuer le roi Olaf, dans la Saga d'Olafur Tryggvason (2013: 275-276), mais celle-ci est imparfaite car Eyvindur Kelda meurt noyé avec ses compagnons, alors que Gaston Lund est mort 
de froid sur l'île déserte de Ketilsey, où le vieillard Jón Ferdinand l'a conduit par erreur.

Au cours de son interrogatoire, Johanna mentionne à son tour deux récits de supplice tirés respectivement de la Saga des vikings de Jómsborg et du Dit d'Ormur Stórólfson. Le premier récit concerne l'exécution manquée de Svein Buáson dont elle rapporte qu'elle a donné naissance à l'épreuve d'initiation des nouveaux membres de l'Association des Vikings du Jómsborg (2013: 312); la doctoresse qualifie d'ailleurs cette épreuve de «cérémonie imbécile» (2013 : 312) et ce jugement est évidemment à mettre en rapport avec le fait que cette pratique a causé la mort de son fiancé, Einar. Un peu plus tard, lorsqu'un des enquêteurs insinue que Johanna s'en est peut-être pris à Kjartan, elle perd patience et demande à l'enquêteur s'il croit qu'elle l'a "attaché à un poteau» et lui a «arraché les tripes ou quelque chose dans le genre » (2013 : 319) ; ainsi que Grímur, le bourgmestre de Flatey, l'explique ensuite aux policiers, il s'agit peut-être d'une allusion à la mort d'Ásbjörn Prúdi, qui est rapportée dans le Dit d'Ormur Stórólfson (2013: 319-320).

Les cas de morts non naturelles dans le roman sont donc systématiquement rattachés, à tort ou à raison, à des épisodes sanglants contenus dans le Livre de Flatey. Ce dernier semble constituer un prisme pour comprendre des évènements à priori incompréhensibles, mais il fonctionne aussi comme une source d'inspiration puisque c'est la Saga des Vikings du Jómsborg qui a entraîné la création de l'association du même nom et de sa dangereuse cérémonie d'entrée, alors que le supplice de l'«aigle de sang », évoqué à plusieurs reprises dans le manuscrit, a été pratiqué par Pormódur Krákur sur le cadavre noyé de Bryngeir afin que le Livre de Flatey revienne en Islande. Lévocation de ces épisodes sert donc avant tout à étayer le récit de l'enquête sur les morts de Gaston Lund et Bryngeir, mais les réponses de Johanna, tout comme, dans une moindre mesure, celles de Sigurbjörn et de Grímur, fournissent également des informations sur le rapport entretenu par certains des personnages avec les textes figurant dans le Livre de Flatey. Par ailleurs, les explications que Sigurbjörn et Grímur donnent aux policiers de Reykjavik sur les récits de morts violentes figurant dans le manuscrit permettent de renseigner les enquêteurs mais aussi le lecteur sur ce thème et l'on peut donc affirmer qu'elles possèdent aussi une dimension didactique.

Les onze citations de titres d'œuvres médiévales islandaises non associées à d'autres formes d'intertextualité concernent huit ouvrages, à savoir la Heimskringla (deux occurrences), la Saga d'Olaf Tryggvason (une occurrence), la Saga de Saint Olaf (une occurrence), la Saga de Sverrir (deux occurrences), la Saga de Håkon l'Ancien (une occurrence), les Annales du Livre de Flatey (une occurrence), la Saga des Orcadiens (une occurrence) et le Dit de Sneglu Hali (deux occurrences). Les deux mentions de la Heimskringla figurent dans les explications que Johanna donne à Kjartan sur le Livre de Flatey avant que tous deux n'entreprennent de résoudre l'«énigme de Flatey » : la doctoresse de 
l'île raconte ainsi que «le Livre de Flatey est une sorte de monstre comparé à la Heimskringla de Snorri Sturluson qui traite une matière semblable» (2013 : 41) et ajoute un peu plus loin que «La Heimskringla de Snorri a probablement dû être un best-seller en Norvège » (2013: 58). Ces deux références, qui figurent dans l'exposé que Johanna improvise sur le Livre de Flatey, ont pour but de fournir des éléments d'information sur l'époque et les circonstances de la création de ce manuscrit en utilisant une œuvre très connue comme point de comparaison.

Juste après la première apparition de la Heimskringla, l'exposé de Johanna contient une énumération assez fragmentaire des textes contenus dans le Livre de Flatey: en effet, elle ne mentionne que cinq œuvres, à savoir la Saga d'Olaf Tryggvason, la Saga de Saint Olaf, la Saga de Sverrir, la Saga de Håkon l'Ancien et les Annales du Livre de Flatey (2013: 42). Malgré le caractère très lacunaire de cette liste, sa fonction est, comme du reste l'ensemble de l'exposé de Johanna, de fournir des informations sur le manuscrit.

La seconde mention de la Saga de Sverrir apparaît lorsque le fermier Sigurbjörn, fin connaisseur du Livre de Flatey, raconte à Kjartan qu'il s'est disputé avec Gaston Lund dans la bibliothèque de l'île au sujet du lieu où doit être conservé le manuscrit et qu'il a posé des questions au professeur danois pour évaluer ses connaissances sur cette saga, sans grand succès, notamment à cause de difficultés linguistiques (2013: 134). Le choix de cette saga comme élément du petit test concocté par Sigurbjörn n'est évidemment pas anodin, puisque la solution de l'« énigme de Flatey» est une phrase contenue dans une strophe insérée au sein de cette saga : «Échappe à la mort constant, y va tout inconstant»; par ailleurs, le mot «constant», qui figure dans cette phrase, est à la fois le mot tracé avec des pierres par Gaston Lund à Ketilsey et... le nom du bateau de Sigurbjörn.

Quelques pages plus loin, Egill, le réceptionniste de l'hôtel Borg à Reykjavik, réussit, à la demande de Dagbjartur, à identifier grâce à une photo de presse l'homme qui l'a interrogé au sujet de Gaston Lund: il s'agit de Friðrik Einarsson, ancien collègue et ami du père de Johanna mais aussi père du fiancé de cette dernière, qui a fait paraître dans un journal une «étude sur les meurtres dans la Saga des Orcadiens» (2013 : 138). Cette mention de la Saga des Orcadiens et des meurtres qu'elle relate doit naturellement être mise en rapport avec le fait que cette œuvre contient notamment, ainsi que Sigurbjörn l'explique aux policiers, une description de l'aigle de sang.

Les deux dernières citations de titre d'œuvre médiévales islandaises non associées à d'autres formes d'intertextualité sont celles du Dit de Sneglu Halli: cette œuvre, qui appartient au genre des paettir, nous raconte l'histoire de Halli, un Islandais à la langue bien pendue, qui entre au service du roi du Norvège Harald Hardrada en tant que scalde (poète) mais se retrouve rapidement en concurrence avec un autre scalde islandais, Thjodolf (Turco, 2015: 194). Lors de son interrogatoire par les policiers, Johanna Thorvald explique que Bryngeir a décidé de la courtiser après avoir lu dans le journal de leur lycée un exposé 
que Johanna avait écrit sur le Dit de Sneglu Halli (Ingólfsson, 2013 : 311) car «il trouvait quelque part affriolant et sexy qu'une lycéenne de dix-huit ans écrive un texte pareil» (2013: 315). À en croire Johanna, sa décision d'écrire un sujet sur ce thème a pourtant une explication des plus prosaïques, puisqu'elle explique à propos du Livre de Flatey:

J'ai utilisé de temps en temps ce livre comme sujet d'exposé au lycée quand je ne voulais pas trop me fatiguer. J'en connaissais la matière tellement bien après avoir écouté mon père donner quantité de conférences en cinq langues que j'étais capable d'écrire un devoir convenable sur n'importe quel sujet contenu dans ce livre.

$(2013: 311)$

À l'intérieur de ce groupe de mentions, on peut clairement distinguer deux sous-ensembles, à savoir les mentions figurant dans la description du Livre de Flatey que Johanna fait pour Kjartan et celles apparaissant à d'autres endroits du roman. En effet, les sept mentions contenues dans l'exposé de la doctoresse ont toutes une fonction didactique alors que les quatre autres mentions fournissent des informations sur le rapport des personnages (ici Johanna, Sigurbjörn et dans une moindre mesure Gaston Lund) aux textes contenus dans le manuscrit. Par ailleurs, la seconde mention de la Saga de Sverrir figure dans le récit de la rencontre de Sigurbjörn et de Gaston Lund, ce qui contribue à faire avancer l'enquête sur les faits gestes et du savant danois à Flatey avant sa mort tragique à Ketilsey, alors que la mention de la Saga des Orcadiens apparaît lors de l'identification de Friðrik Einarsson, qui est un ami proche des Thorvald et connaît également Gaston Lund, ce qui fait de lui un témoin précieux pour le policier Dagbjartur.

Ainsi que nous l'avons mentionné ci-dessus, L'Énigme de Flatey contient une seule citation de genre littéraire et celle-ci concerne les sagas des Islandais: en effet, lors d'une conversation à propos des elfes et de leur existence, le sacristain Pormódur dit à Kjartan que les gens «doutent trop » et «doivent croire ce que racontent les sagas d'Islandais [sic] et la Bible, et ce que les anciens transmettent» afin que leurs rêves et leurs souhaits se réalisent (2013: 89). Cette mention des sagas des Islandais fait donc référence au rapport que le sacristain entretient avec les textes anciens et prouve que cette relation se caractérise par une très forte croyance dans les récits des sagas, auxquels il semble prêter des pouvoirs presque magiques.

La première des trois allusions onomastiques créées à partir de noms de personnages et/ou d'auteurs de sagas intervient lorsque le sacristain Pormódur lit dans cette œuvre un chapitre La Saga des frères jurés qui parle de son homonyme, le scalde et guerrier Pormódur Kolbrúnarskáld ${ }^{8}$ (2013 : 74); le sacristain,

\footnotetext{
${ }^{8}$ C'est-à-dire «scalde de Kolbrún (prénom féminin)».
} 
visiblement fier de cette homonymie, y fait lui-même référence en une occasion plus loin dans le récit (2013: 256). Le second clin d'œil est le pseudonyme choisi par Gaston Lund pour s'inscrire à l'Hôtel Borg : le chercheur danois choisit ainsi de s'appeler... Egil Sturluson, soit une combinaison du prénom du héros de la Saga d'Egil, fils de Grimr le Chauve et du nom du père ${ }^{9}$ de son auteur, Snorri Sturluson. L'orthographe inhabituelle du prénom Egil retient d'ailleurs l'attention du réceptionniste de lui, qui porte lui aussi ce prénom, mais dans sa version moderne, c'est-à-dire avec deux «1» (2013: 113). La dernière allusion onomastique intervient lorsque le père de Johanna, Björn Snorri Thorvald, qui est mourant, dit qu'il pense bientôt rejoindre par son homonyme, Snorri Sturluson, dont il affirme qu'il a écrit la Saga de Njáll le Brûlé (2013 : 168).

Ces trois éléments ne contribuent pas réellement à la progression de l'intrigue, ce qui suggère que leur fonction est d'un autre type. Ainsi, la référence à Snorri Sturluson comme auteur de la Saga de Njáll le Brûlé peut être considérée comme possédant une fonction didactique : cette dimension semble moins présente dans l'allusion à l'homonymie entre le sacristain et le scalde Pormódur, mais ce passage constitue en revanche une source d'information supplémentaire sur le rapport que bormódur Krákur entretient avec les textes anciens (la Saga des frères jurés fait d'ailleurs partie des sagas des Islandais). La mention du pseudonyme inventé par Gaston Lund ne possède quant à elle aucune dimension didactique et suppose au contraire de savoir que Snorri Sturluson a écrit la Saga d'Egil, fils de Grimr le Chauve; il s'agit donc davantage d'une plaisanterie pour initiés qui requiert une certaine connaissance de la littérature islandaise médiévale.

L'analyse des fonctions des cas d'intertextualité dans L'Énigme de Flatey permet de constater qu'ils remplissent essentiellement trois grandes fonctions, mais il convient de souligner que certaines références peuvent avoir plusieurs fonctions. On remarque ainsi une cinquantaine d'occurrences où la fonction des cas d'intertextualité est essentiellement de faire progresser l'intrigue, en particulier en ce qui concerne la résolution de l'« énigme de Flatey» et l'enquête sur les morts de Gaston Lund et de Bryngeir, mais on constate aussi l'apparition d'une dimension didactique dans une quinzaine de cas; enfin, l'élément intertextuel fournit des informations sur le rapport entre les personnages et les textes islandais anciens, en particulier ceux contenus dans le manuscrit, dans une petite quinzaine d'occurrences.

${ }^{9}$ En Islande, l'usage du nom de famille tel que nous le connaissons est extrêmement rare; la tradition veut que les Islandais portent le prénom de leur père suivi de la marque du génitif et du suffixe -son (fils) ou -dottir (fille). 


\section{Le rôle de l'objet-manuscrit}

Ces trois fonctions, que l'on peut qualifier de «narrative», «didactique» et «biographique», apparaissent également dans les références au Livre de Flatey en lui-même. Ainsi, au-delà du fait que les textes contenus dans ce manuscrit constituent la clé de l'«énigme de Flatey», la question de son lieu de conservation est également évoquée à plusieurs reprises, et le désaccord entre le professeur Lund, fermement opposé à une éventuelle restitution de ce manuscrit à l'Islande, et certains insulaires de Flatey, chauds partisans d'un retour du Livre de Flatey dans le pays où il a été réalisé, est d'ailleurs brièvement évoqué comme cause possible du destin tragique du savant danois, mais cette hypothèse est écartée assez rapidement. La dimension didactique apparaît essentiellement à travers l'exposé que Johanna fait pour Kjartan dans le prologue de récit de la résolution de l'«énigme de Flatey», où la doctoresse fournit beaucoup d'informations sur la genèse du manuscrit et donne même des détails techniques sur sa fabrication, par exemple sur les matériaux employés $(2013: 75,96)$ ou le type d'écriture (2013 : 105-106).

Bien que seuls trois personnages, à savoir Gaston Lund, Bjorn Snorri Thorvald et sa fille Johanna, aient réellement eu accès au Livre de Flatey, le roman contient de nombreuses informations sur le rapport que les personnages entretiennent avec le manuscrit. Ainsi, plusieurs insulaires de Flatey sont de farouches partisans de la restitution des manuscrits à l'Islande et les habitants veillent également avec un grand soin mâtiné de fierté à l'édition du Livre de Flatey conservée à la bibliothèque mais aussi aux feuilles de l'énigme, qui se trouvent au même endroit et qu'une superstition locale interdit d'ailleurs de transporter hors du bâtiment. L'un des habitants de l'île, le sacristain Pormódur Krákur, va même encore plus loin puisqu'il dépose le cadavre de Bryngeir sur une tombe du cimetière local et lui taille un aigle de sang dans le dos afin de favoriser le retour du Livre de Flatey en Islande, selon un conseil donné par Bryngeir lui-même après que le sacristain lui eut raconté un de ses rêves (2013: 347-349).

Si les insulaires de Flatey sont partisans d'une restitution du manuscrit à l'Islande et que Gaston Lund s'y oppose formellement, Björn Snorri Thorvald est, quant à lui, selon son ami Friðrik Einarsson, «l'un des rares Islandais qui n'avaient absolument aucun avis quant à l'endroit où les manuscrits devaient être conservés. Il voulait seulement les savoir en lieu sûr et y avoir accès facilement...» (2013 : 211). L'ami de Björn Snorri Thorvald explique également que celui-ci avait une démarche scientifique originale: «Au lieu de se focaliser sur le texte, il commença à se faire une idée des copistes» (2013: 212). Johanna y fait également allusion lors de sa rencontre avec Kjartan dans la bibliothèque de Flatey puisqu'elle dit que son père faisait partie des rares personnes qui pou- 
vaient, grâce au manuscrit, entrer en contact avec les Islandais du XIVe siècle (2013: 230).

En plus de ses grandes connaissances sur la genèse du manuscrit, Johanna est la seule à évoquer la valeur esthétique et artistique du livre; dans son exposé sur le manuscrit, elle affirme ainsi que la richesse de ses enluminures l'a sauvé de la destruction (2013 : 93), et lorsque Kjartan la questionne sur ce qu'elle a ressenti en se trouvant en présence du manuscrit, elle répond:

[...] ce livre est le plus beau qu'il m'ait été donné de voir de mes yeux. Ces lettres couleurs anthracite sur un parchemin beige sont comme d'interminables rangées de perles dans un collier. Les enluminures sont pour moi les plus jolies fresques des voûtes du palais d'un roi.

$(2013: 231)$

\section{Le rapport des personnages au Livre de Flatey}

L'analyse des passages concernant le rapport que les personnages entretiennent avec le Livre de Flatey et les textes qu'il contient fait aisément apparaître l'existence de deux grands groupes, à savoir celui des "connaisseurs » et celui des «ignorants», mais il convient de souligner que ces deux groupes, loin d'être homogènes, se composent de plusieurs sous-groupes et cas particuliers. Au sein du groupe des «connaisseurs», on peut distinguer les savants, les insulaires de Flatey, les personnes possédant un rapport davantage symbolique, voire spirituel au manuscrit et à son contenu et Johanna Thorwald, qui ne rentre dans aucune de ces catégories.

Le groupe des savants est composé de Gaston Lund, de Björn Snorri Thorvald ainsi que de Friðrik Einarsson, l'ami du professeur Thorvald, et du poète et historien Árni Sakárias, qui raconte l'histoire de l'«énigme de Flatey» à Dagbjartur, le premier policier de Reykjavik chargé d'enquêter sur la mort du professeur Lund. Il convient toutefois de souligner que la relation de Friðrik Einarsson et d'Árni Sakárias avec le manuscrit n'est que légèrement esquissée, ce qui est sans doute à mettre en rapport avec le fait qu'il s'agit de deux personnages relativement secondaires du roman. Dans le cas de Gaston Lund, le récit rapporte qu'il est un farouche opposant à la restitution du Livre de Flatey et d'autres manuscrits anciens à l'Islande (2013: 151-152), car il affirme que ceux-ci y seraient conservés dans de moins bonnes conditions qu'à Copenhague et seraient moins accessibles, ce qui lui vaut l'inimitié de ses collègues islandais; à Flatey, le pasteur Hannes et l'agriculteur Sigurbjörn lui font également part de leur désaccord avec lui à ce sujet $(2013$ : 101, 134). Le pasteur raconte également 
à Kjartan que le professeur Lund voulait résoudre l'«énigme de Flatey», car il était désireux d'obtenir une meilleure place dans le restaurant où se réunissent les membres de l'Académie des Sciences de Copenhague (2013 : 102-103). Au sein de ce groupe, Björn Snorri Thorvald se distingue à la fois par sa neutralité dans le débat où doivent être conservés les manuscrits et aussi par le thème de ses recherches sur le Livre de Flatey, puisqu'à l'inverse de ses collègues, qui se concentrent essentiellement sur le contenu des textes, il s'intéresse davantage à la genèse du manuscrit et à ses copistes. Après son renvoi du Danemark et son arrivée sur l'île de Flatey, il essaie cependant de trouver la solution de l'énigme du même nom et il n'est d'ailleurs pas loin d'y parvenir, mais ses efforts l'épuisent et accentuent les effets de la maladie qui lui fut fatale.

Les insulaires de Flatey forment un groupe assez homogène, qui se caractérise notamment par sa bonne connaissance du manuscrit portant le nom de leur île; ainsi, lorsque Kjartan demande à Grímur qui sont les habitants capables de renseigner les voyageurs sur l'histoire du manuscrit, le bourgmestre répond:

Tout un chacun [...]. La plupart des insulaires sont capables de récapituler son histoire si on le leur demande. Sigurbjörn de Svalbarði est très cultivé et a souvent recours à ce livre, mais le révérend Hannes est meilleur en danois et est capable de s'entretenir avec les étrangers.

$(2013: 65)$

Grímur ne fait d'ailleurs pas exception à la règle, puisque même s'il affirme ne pas avoir «tout le livre en tête comme [son] concitoyen Sigurbjörn de Svalbarði » (2013 : 319), il est tout de même capable d'expliquer aux enquêteurs l'allusion de Johanna au meurtre d'Ásbjörn Prúdi dans le Dit d'Ormur Stórólfson (2013 : 319-320). Quant à Sigurbjörn, il se permet même de tester les connaissances de Gaston Lund sur la Saga de Sverrir, de comparer les décès de Gaston de Lund et de Bryngeir avec certains épisodes du Livre de Flatey mais aussi de conseiller aux policiers qui l'interrogent de lire cette œuvre! L'attitude de Sigurbjörn est assez représentative de celle de la majorité des insulaires, qui non seulement connaissent bien l'ouvrage mais éprouvent un certain sentiment de fierté à son égard ainsi qu'une forme de mépris envers les spécialistes, qu'ils taxent souvent d'ignorance et d'arrogance: Hallbjörg, la vieille dame qui garde la clé de la bibliothèque dit ainsi des visiteurs qui veulent résoudre l'énigme que ce sont des «m'as-tu vu qui prétendent connaître Le livre de Flatey» (2013 : 319). La seule exception à ce jugement concerne Björn Snorri Thorvald et sa fille, qui sont installés sur l'île et sont appréciés par les habitants. Les insulaires reprochent aussi aux visiteurs de ne pas respecter leur patrimoine: Sigurbjörn rapporte ainsi le vol des feuilles de l'énigme par Bryngeir et le fait que Kjartan ait oublié de fermer à clé la porte de la bibliothèque en pestant contre «ces intellectuels de Reykjavik » qui «ne sont même pas fichus de faire attention aux objets de valeur» $(2013: 274)$. 
Parmi les connaisseurs du Livre de Flatey, le rapport du journaliste Bryngeir - fils caché de Gaston Lund et responsable de la mort du fiancé de Johanna - au manuscrit est assez singulier. L'examen de cette relation permet tout d'abord de constater la présence d'un intérêt d'ordre intellectuel: ainsi, Bryngeir présente le Livre de Flatey comme sa «vieille lecture favorite» (2013: 170), décide de courtiser Johanna après avoir lu son travail sur le Dit de Sneglu Halli dans le journal de l'établissement (2013: 311) et éprouve une certaine admiration envers les auteurs du manuscrit, qu'il qualifie de «génies» (2013: 171). En revanche, le nom du manuscrit lui déplait, car il «trouve très pénible que ce joyau porte le nom d'un îlot insignifiant rien que parce qu'il a été conservé à cet endroit sous un toit pouilleux pendant plusieurs décennies » (2013: 171). Le rapport de Bryngeir au Livre de Flatey est également empreint d'un certain symbolisme, voire même d'une dimension spirituelle, comme le suggère sa participation à l'«Association des Vikings du Jómsborg » et surtout le fait qu'il conseille au sacristain Pormódur Krákur de tailler un aigle de sang dans le dos d'un mort et de le déposer sur une tombe du cimetière de l'île afin que le Livre de Flatey revienne en Islande (2013: 347-349).

Le sacristain Pormódur Krákur est d'ailleurs le seul habitant de l'île à avoir une opinion positive de Bryngeir, dont il vante le talent pour interpréter les rêves (2013 : 278). Comme le fils caché de Gaston Lund, le sacristain, mêle volontiers littérature et spiritualité, ainsi que le prouvent sa remarque à propos de la nécessité de croire les sagas des Islandais et surtout le fait qu'il a taillé un aigle de sang dans le dos du cadavre de Bryngeir pour que le Livre de Flatey revienne en Islande. Le rapport très particulier que Pormódur Krákur entretient avec le manuscrit et les textes qu'il contient est à mettre en relation avec le caractère du sacristain, dont le bourgmestre dit qu'il «croit à plus de choses qu'il n'est courant de le faire» $(2013: 65-66)$.

Au-delà de ses connaissances sur le manuscrit, qui sont très complètes, Johanna est certainement le personnage du roman dont le rapport au manuscrit est le plus riche, puisqu'elle en vante notamment la beauté et compare les pages du livre à de vieux amis $(2013: 231)$ mais évoque également ses sentiments contrastés vis-à-vis de certains des textes qu'il contient: la fille de Björn Snorri Thorvald explique ainsi qu'elle apprécie peu les personnages historiques du manuscrit et ajoute que «la plupart était des ordures de la pire espèce» avant d'affirmer son admiration envers la sagesse des annalistes islandais du Moyen Âge (2013 : 71). La relation que Johanna entretient avec le Livre de Flatey est également la seule à évoluer avec le temps: elle commence ainsi quand son père se met à lui lire des passages du manuscrit alors qu'elle est encore enfant (2013: 46), puis, peu après, elle se met à raconter à sa manière ses histoires favorites (2013: 47). Par la suite, le rapport de Johanna au manuscrit devient plus pragmatique, notamment lorsque, en profitant des connaissances acquises grâce aux conférences et aux explications de son père, elle utilise le Livre de Flatey comme sujet d'exposé au 
lycée pour ne «pas trop [se] fatiguer» (2013:311); devenue adulte, elle continue d'éprouver un fort attachement pour le manuscrit et c'est grâce à son aide précieuse que Kjartan peut résoudre l'« énigme de Flatey».

Le groupe des «ignorants» se compose essentiellement de quatre personnages: trois d'entre eux, à savoir Kjartan, bórólfur et Lukás sont originaires de Reykjavik et viennent à Flatey pour enquêter sur les décès qui y ont lieu alors que le dernier, le jeune Benni, habite sur l'île. Il convient cependant de signaler que parmi les deux policiers de Reykajvik, Lukás prend très peu la parole et jamais au sujet du manuscrit : le point de vue exposé est donc celui de l'enquête Pórólfur, mais le silence du second policier tend à indiquer qu'il n'en sait pas plus long sur le manuscrit. Le troisième policier, Dagbjartur, mène, quant à lui, l'enquête dans la capitale islandaise, se renseigne sur «l'énigme de Flatey» et remarque, tout comme le réceptionniste de l'hôtel Borg, l'orthographe inhabituelle du prénom «Egil» utilisé par Gaston Lund dans son pseudonyme, mais sa réponse assez vague («Oui, je comprends que ce nom ait attiré ton attention», 2013: 113) ne permet pas de déterminer s'il a compris la construction de ce nom d'emprunt.

Malgré sa participation à l'« Association des Vikings du Jómsborg» Kjartan ne possède pas une connaissance très approfondie du Livre de Flatey avant de venir dans l'île du même nom: ainsi qu'il le précise aux policiers de Reykjavik, il est devenu membre de cette association à cause de son penchant pour la « gloriole», de son goût pour la littérature contemporaine internationale et parce qu'il aimait bien faire la fête à l'occasion (2013: 329-330). Durant son séjour à Flatey, Johanna Thorvald lui raconte, à sa demande, la genèse et la signification de ce manuscrit et l'aide à résoudre l'«énigme de Flatey» à partir des réponses de Gaston Lund et de Björn Snorri Thorvald.

Le fait que l'enquêteur Pórólfur ne connaît pas le Livre de Flatey est révélé par un savoureux dialogue entre Pórólfur et Sigurbjörn lors de l'interrogatoire de ce dernier: lorsque l'insulaire s'étonne de ce qu'il lui semble être une grave lacune de culture générale, le policier répond sans ménagement que le manuscrit «ne fait pas partie des lectures obligatoires pour les policiers» (2013:275). Pórólfur fait toutefois preuve d'une certaine ouverture d'esprit puisque lorsque Sigurbjörn lui explique que les morts de Gaston Lund et de Bryngeir présentent des analogies avec celles de plusieurs épisodes figurant dans les œuvres contenues dans le Livre de Flatey, le policier demande si le livre est long, puis il demande au bourgmestre s'il peut lui procurer une édition de cet ouvrage (2013 : 276). Grímur s'acquitte d'ailleurs rapidement de cette tâche, car le sacristain Pormódur Krákur amène son exemplaire personnel lors de son interrogatoire pour le prêter au policier (2013 : 278). Les bonnes dispositions de Pórólfur semblent cependant de courte durée car lorsque Grímur doit lui expliquer l'allusion de Johanna au meurtre d'Ásbjörn Prúdi et précise que cet épisode se trouve dans le Livre de Flatey, il lâche un «Encore ce foutu livre?» qui témoigne d'une certaine exaspération $(2013: 319)$. 
La méconnaissance du Livre de Flatey par les deux policiers envoyés dans l'île pour enquêter sur les décès de Gaston Lund et de Bryngeir semble pouvoir être rapprochée d'un épisode figurant dans un autre roman islandais, à savoir Les Anges de l'univers d'Einar Már Guðmundsson. En effet, dans ce livre, un personnage raconte comment, un soir, lui et deux amis ont trouvé une pioche sur un chantier de construction puis ont rencontré des policiers qui leur ont demandé ce qu'ils comptent en faire. Rognvald, le porteur de l'outil répond alors: «tuer un homme et un autre », (hoggva mann ok annan), ce qui constitue le dernier vers d'une strophe contenue par la Saga d'Egill fils de Grímur le Chauve et dont la création est attribuée au héros éponyme du récit, qui l'aurait composée pour célébrer son premier meurtre à l'âge de sept ans, mais les policiers, qui n'ont visiblement pas saisi la référence littéraire, interprètent cette réponse au premier degré, arrêtent les trois hommes et les font monter dans le panier à salade. Alors qu'ils roulent vers le commissariat, Rognvald enjoint vertement aux policiers de rentrer chez eux lire les sagas, mais l'un des agents dit que cela n'est pas nécessaire (Guðmundsson, 1998; cité par Turco, 2015 : 185-186).

Bien que les romans L'Énigme de Flatey et Les Anges de l'univers décrivent tous deux des policiers assez peu compétents en littérature islandaise ancienne, il n'est pas certain que ces personnages remplissent exactement la même fonction dans les deux récits. De fait, Jeffrey Turco considère davantage l'épisode du malentendu présent dans le roman d'Einar Már Guðmundsson comme la preuve de l'existence au sein de la société islandaise de deux communautés, qu'il qualifie respectivement d'« exotérique» et d'«ésotérique» en fonction de leur façon d'interpréter les sagas; selon Turco, les autorités s'efforceraient de discipliner les membres de cette seconde communauté «à cause d'un penchant pour des références intertextuelles spirituelles et apparemment inoffensives qui toutefois sapent subtilement la hiérarchie sociale» (2015: 186-187). Cette interprétation ne fonctionne cependant pas aussi bien dans le cas des policiers de L'Énigme de Flatey: si les enquêteurs sont effectivement raillés par Sigurbjörn pour leur méconnaissance du Livre de Flatey et ont besoin de l'aide d'autres personnages pour comprendre les allusions à cette œuvre, la communauté des connaisseurs du livre, et plus particulièrement sa composante insulaire, ne manifeste pas de désir de renverser l'ordre social et semble seulement exprimer sa désapprobation vis-à-vis du recul des traditions et du manque d'intérêt pour la culture islandaise séculaire. Dans ce contexte, les policiers paraissent davantage symboliser le monde citadin, ouvert à la modernité et peu intéressé par le patrimoine culturel national, mais il convient de signaler que ce rôle ne leur est pas exclusivement dévolu: ainsi, le jeune Benni, malgré le fait qu'il habite sur l'île de Flatey, est davantage intéressé par Elvis Presley que par le Livre de Flatey.

Grand admirateur d'Elvis, de Ben Hur et de la culture américaine, Benni est en effet le seul insulaire de Flatey qui ne semble pas du tout intéressé par le manuscrit : il s'étonne ainsi de l'engouement de Bryngeir pour le Livre de Flatey 
et explique qu'il a essayé de lire mais l'a trouvé «barbant» avant d'ajouter qu'il a été un peu dérouté par son orthographe (Ingólfsson, 2013: 170). Lorsque le journaliste s'épanche sur l'importance du manuscrit et critique le nom retenu pour le désigner, Benni répond qu'il n'a pas d'opinion sur la question et fait la vague promesse de lire le Livre de Flatey à l'avenir, mais cette résolution semble surtout destinée à amadouer Bryngeir afin qu'il lui redonne un peu de rhum (2013: 171).

\section{Conclusion}

L'analyse de la place et de l'usage du Livre de Flatey ainsi que des textes islandais médiévaux anciens dans L'Énigme de Flatey de Viktor Arnar Ingólfsson permet de constater que la plupart des cas d'intertextualité proviennent d'œuvres contenues dans ce manuscrit: plus de la moitié de ces références figurent ainsi dans le récit de la résolution de l'«énigme de Flatey», ce qui prouve que leur fonction principale est de contribuer à l'avancée de l'intrigue. Par ailleurs, un nombre significatif de cas d'intertextualité possèdent une dimension didactique ou bien permettent d'éclairer le lecteur sur le rapport que les personnages entretiennent avec le manuscrit et les textes qu'il contient. Les différents types de relations décrites contribuent à proposer une division en deux grands groupes, dont le premier contient ceux des Islandais qui attachent de l'importance au Livre de Flatey et aux textes anciens, alors que la seconde rassemble ceux qui y sont indifférents.

Cette division binaire n'est d'ailleurs pas spécifique à L'Énigme de Flatey de Viktor Arnar Ingólfsson mais figure également dans un autre roman islandais contemporain construit autour du destin d'un célèbre manuscrit médiéval islandais, à savoir Le Livre du roi d'Arnaldur Indridason ${ }^{10}$, qui présente de nombreuses similitudes avec le roman mentionné dans cet article. Ainsi, les intrigues des deux romans se situent dans la même temporalité, puisqu'elles ne sont chronologiquement éloignées que de cinq ans (les deux récits se déroulent respectivement en 1960 et en 1955) et se placent toutes les deux dans l'intervalle de vingt-sept ans entre l'indépendance de l'Islande en 1944 et la restitution des manuscrits par le Danemark en 1971. La distinction opérée entre les personnages en fonction de leur relation des individus aux manuscrits et aux textes anciens s'étend également à leur rapport au passé et à leur attitude du monde extérieur : ainsi, les personnages connaissant bien les manuscrits démontrent souvent un

${ }^{10}$ Arnaldur Indridason, Le Livre du roi, traduit par Patrick Guelpa, Paris, Éditions Métaillé, 2013. Texte original: Konungsbók. Forlagid, Reykjavík, 2006. 
attachement aux traditions et au patrimoine de l'Islande alors que ceux qui ne s'intéressent pas à ces œuvres sont souvent des porte-paroles de la modernité et de l'ouverture aux influences extérieures et notamment à la culture américaine. La différence d'attitude vis-à-vis des manuscrits semble donc refléter les lignes de clivage qui traversent la société islandaise durant les premières décennies suivant le retour à l'indépendance en 1944, et se manifestent notamment par des attitudes très contrastées au sujet de l'existence de la base aérienne de l'OTAN à Keflavik (Ingimundarson, 2011).

Cette dimension binaire, également présente dans Le Livre du roi, semble aussi se retrouver dans la réception des textes médiévaux par les lecteurs; ainsi le fait que les cas d'intertextualité aient des fonctions essentiellement pragmatiques suggère que la plupart de ces occurrences ont un seul niveau de lecture alors que la présence de nombreux éléments revêtant une fonction didactique tend à indiquer que le «lecteur implicite» (Iser, 1985:60-76) du roman ne doit pas nécessairement posséder une connaissance très approfondie de la littérature islandaise médiévale pour comprendre ce récit, mais plusieurs cas précis, comme les proverbes cités par Johanna, les paraphrases d'épisodes non accompagnées de citation de titres dans les réponses à «l'énigme de Flatey » ou le pseudonyme d'«Egil Sturlurson» utilisé par Gaston Lund, nécessitent un savoir plus étendu pour en saisir totalement le contexte, ce qui semble supposer que ces passages précis peuvent être destinés à des lecteurs davantage connaisseurs des textes produits dans l'Islande du Moyen Âge. Une telle division rappelle donc non seulement celle présente parmi les personnages du roman, mais elle évoque aussi dans une certaine mesure celle postulée par Jeffrey Turco (2015 : 186-187); malgré l'existence de ces analogies dignes d'intérêt, il convient toutefois de souligner que ces résultats sont fondés sur l'analyse d'œuvres littéraires et ne sauraient se substituer à une enquête de fond sur le rapport que les Islandais entretiennent avec les plus anciens textes de leur littérature nationale.

\section{Bibliographie}

\section{Ouvrage étudié :}

Ingólfsson V. A., 2013 : L’Énigme de Flatey. Trad. P. Guelpa. Seuil, Paris. Texte original : Flateyjargáta. Mál og menning, Reykjavík, 2002.

Autres œuvres littéraires évoquées :

Guđmundsson E. M., 1998: Les Anges de l'univers. Trad. C. Eyjólfsson. Flammarion, Paris. Texte original: Englar alheimsins. Almenna bókafélagið, Reykjavík, 1993.

Indridason A., 2013: Le Livre du roi. Trad. P. Guelpa. Éditions Métaillé, Paris. Texte original : Konungsbók. Forlagid, Reykjavík, 2006. 


\section{Ouvrages de références :}

Genette G., 1982 : Palimpsestes. La littérature au second degré. Seuil, Paris.

Ingimundarson V., 2011: The Rebellious Ally: Iceland, the United States, and the Politics of Empire 1945-2006. The Republic of Letters, Dordrecht.

Iser W., 1985 : L'acte de lecture. Mardaga, Bruxelles.

Lethbridge E., 2014 : Hvorki glansar gull á mér/ né glaestir stafir i línum: A survey of Medieval Icelandic Íslendingasögur Manuscripts and the case of Njáls saga. "Arkiv för nordisk filologi", no 129, p. 55-89.

Morawiec J., 2018: Interdisciplinarity of research on the Jómsborg legend. "Questiones Medii Aevi Novae", n² 23, p. 125-138.

Turco J., 2015: Loki, Sneglu-Halla páttr, and the case for a skaldic prosaics. In: New Norse Studies. Ed. J. Turco. Cornell University Library, Ithaca, p. 185-241.

\section{Notice bio-bibliographique}

Docteur en Histoire du Moyen Âge, adjoint au Département d'Histoire de l'Institut d'études slaves, membre du projet Testimonia sur les sources écrites concernant l'histoire des Slaves jusqu'en 1200 et membre de la Commission Internationale Gerard Labuda sur l'ethnogenèse des Slaves. Intérêts scientifiques: histoire culturelle de l'Europe Centrale au haut Moyen Âge (900-1300), production historiographique en Europe Centrale au Moyen Âge, contacts culturels entre la France et l'Europe Centrale au Moyen Âge, numismatique de l'Europe Centrale au haut Moyen Âge, médiévalisme. Principale publication: Annales Magdeburgenses Brevissimi. The Short Annals of Magdeburg and their signification in the discussion on the genesis of Czech and Polish annalistic productions. Olomouc, Vydavatelství Filozofické Fakulty Univerzity Palackého v Olomouci, Collection Historie, t. 5, 2016.

adrien.queret-podesta@ispan.waw.pl 